\title{
From Local Measurements to an Upscaled Capillary Pressure-Saturation Curve
}

\author{
S. Bottero · S. M. Hassanizadeh · P. J. Kleingeld
}

Received: 1 March 2010 / Accepted: 26 January 2011 / Published online: 3 March 2011

(C) The Author(s) 2011. This article is published with open access at Springerlink.com

\begin{abstract}
Capillary pressure-saturation relationship plays an important role in the description of two-phase flow in porous media. Commonly, this relationship is determined in laboratory on a sample of few centimeters and it is then used in numerical modeling of two-phase in domain sizes of hundreds to thousands of meters. The correctness of such approach has been hardly ever questioned. In this study, an upscaled capillary pressure is determined from local pressure and saturation measurements employing a rigorous averaging procedure. Drainage and imbibition experiments were performed in a column of 21 $\mathrm{cm}$ long. The experiments were performed as a series of equilibrium steps; each time we changed the boundary pressures incrementally and then waited until an equilibrium distribution of fluids was reached. Phase pressures and saturation inside the column as well as external pressure and average saturation were recorded at each equilibrium step. Various averaging operators were considered: simple average, simple phase-average, intrinsic phaseaverage, and centroid-corrected average. Also, a potential-based average operator was introduced as reference curve to establish which operator gives the correct average pressure. Large differences were found for the average non-wetting phase pressure using different operators during primary drainage. However, when both phases were present throughout the domain (e.g. during main drainage) the differences between pressures obtained by various average operators were negligible. In such cases, the centroids of the two phases and the centroid of the averaging domain were close to each other. The comparison between averaged capillary pressure-saturation curves has shown that the centroid-corrected averaging operator is the most appropriate operator.
\end{abstract}

S. Bottero $(\varangle) \cdot$ S. M. Hassanizadeh · P. J. Kleingeld

Department of Earth Sciences, Utrecht University, P.O. Box 80021, 3508 TA Utrecht, The Netherlands

e-mail: bottero@geo.uu.nl

Present Address:

S. Bottero

Department of Biotechnology, Delft University of Technology, Julianalaan 67,

2628 BC Delft, The Netherlands 
Keywords Two-phase flow experiments $\cdot$ Average pressure $\cdot$ Capillary pressure-saturation relationship

\section{Introduction}

Capillary pressure-saturation relationship plays a central role in the mathematical modeling of two-phase flow in porous media. For a given porous medium, this relationship is commonly determined from laboratory experiments on samples which are a few centimeters in length. The resulting curve is subsequently used in the (numerical) modeling of two-phase flow in domains of various dimensions, from laboratory columns to oil fields. The correctness of such a practice has been hardly ever questioned. In fact, in the numerical modeling of two-phase flow in porous media, two important length scales are encountered; these are related to the modeling domain size and the numerical grid size. There exists another length scale, related to the size of measurement windows of material properties. Given the fact that modeling parameters are specified as constants over a numerical grid, they should be measured over length scales comparable to grid sizes.

Therefore, for example, for a laboratory column of tens of centimeters in length, the capillary pressure-saturation curve should be determined from measurements with a length scale of a few millimeters, which is the typical size of grid cells when modeling such a column. At the same time, the measurement window must be much larger than typical pore sizes. For field applications, however, e.g. when modeling the spread of organic liquids in soil, model parameters should be determined at the scale of a few tens of centimeters to one meter, which is the typical grid size for field-scale simulations. This time, the measurement window must be much larger than the typical size of micro heterogeneities (e.g. aggregates, lenses, and fissures, etc.).

However, if it is commonly not possible to measure two-phase flow parameters directly at the scale of, say $1 \mathrm{~m}$, because usually measurement sensors of such dimensions are not available. One should be able to average parameter values obtained at a small scale and obtain corresponding larger-scale parameters. In fact, there are a number of studies where upscaling of capillary pressure-saturation curves has been carried out (see Quintard and Whitaker 1988; Ahmadi and Quintard 1994; Desbarats 1995; Ataie-Ashtiani et al. 2001, 2002; Das et al. 2004; Manthey et al. 2005; Eichel et al. 2005; Gielen et al. 2004, 2005; Gielen 2007; Korteland et al. 2009). These works, however, are all computational in nature. Up to now, to the best of our knowledge, no upscaling of measured capillary pressure-saturation curves has been performed. We recognize that upscaling may also involve changes in governing equations. Here, we assume that two-phase flow equations remain the same when we go from the scale of $1 \mathrm{~cm}$ to, say, $20 \mathrm{~cm}$. Therefore, only material properties need to be averaged properly in order to obtain upscaled parameters.

The upscaling of capillary pressure is closely related to the averaging of fluid pressures, which itself has been the subject of a number of recent studies (see Nordbotten et al. 2007, 2008; Korteland et al. 2009). The question is how should one average the pressure. In the literature on averaging, macroscale pressure is always defined as the intrinsic phase-average of the microscale pressure, where basically the pressure of a phase is weighted by its volume fraction. This definition, however, has been shown to lead to incorrect results. For example, Nordbotten et al. (2007) averaged the microscale single-phase flow equation for cases with a porosity gradient and showed that the classical definition of average pressure (the i.e. intrinsic phase-volume average, see Eq. 6) does not lead to the classical Darcy's law, but gives rise to a non-physical gravity term. In a second article, Nordbotten et al. (2008) 
studied the averaging of two-phase flow in porous media and showed that, even in the case of homogeneous porous media, the intrinsic phase-volume average pressure leads to additional terms in Darcy's law. In this article, we present results of a study involving measurement of local-scale fluid pressures along a soil column and averaging of results to the column scale.

Four different averaging operators are used to obtain various column-scale average pressures. Based on the requirement of a hydrostatic pressure distribution under no-flow conditions, we have determined which definition of average pressure physically is acceptable. The average pressure values have been used together with average saturation to construct column-scale capillary pressure-saturation curves.

\section{Experimental Set-up}

An experimental set-up was designed to allow performing both drainage and imbibition experiments. Details of the set-up can be found in Bottero (2009). A schematic representation of the set-up is shown in Fig. 1. It consisted of a plexiglas column for holding soil, an inflow reservoir and an outflow reservoir. These reservoirs were made of glass burettes, each $120 \mathrm{~cm}$ high and $4.95 \mathrm{~cm}$ in inner diameter. The plexiglas column was $21 \mathrm{~cm}$ high and had an inner diameter of $9.83 \mathrm{~cm}$. It consisted of a flow distribution plate at the bottom, a stainless-steel porous plate (placed on the distribution plate) for supporting the sand, the sand chamber, another stainless-steel porous plate for keeping the sand in place, and an outflow chamber (see Fig. 2). Tetrachloroethylene (PCE) and de-mineralized, de-aired water were used as the non-wetting and wetting phases, respectively. Their physical properties are given in Table 1. To visualize the displacement of one phase by the other, PCE was colored with Sudan-Red dye $(1,3 \mathrm{mg} / \mathrm{l})$. We could clearly see that there was no fingering at any time (drainage or imbibition) and there was a macroscopically sharp front. This shows the soil was quite homogeneous.

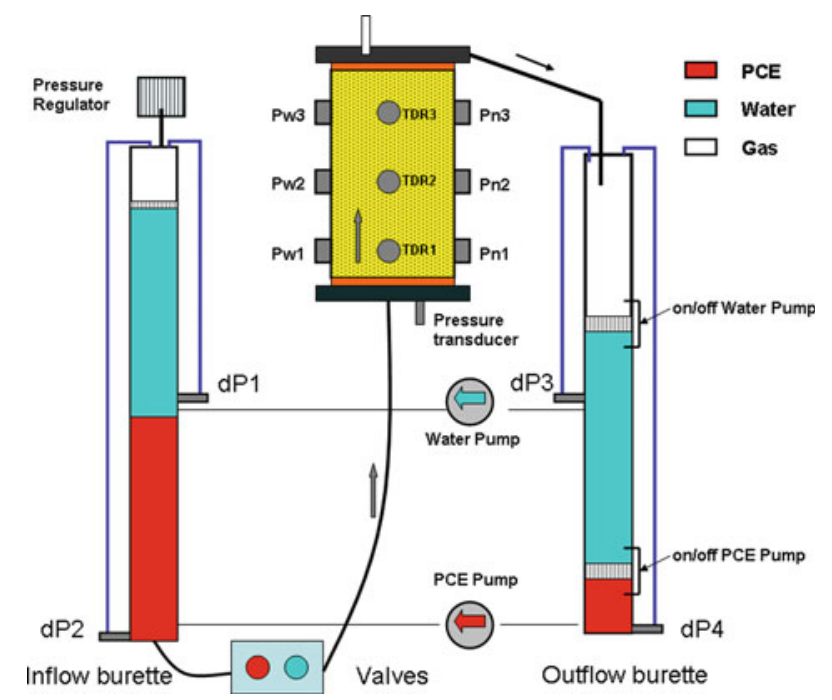

Fig. 1 Experimental set-up (not to scale) 


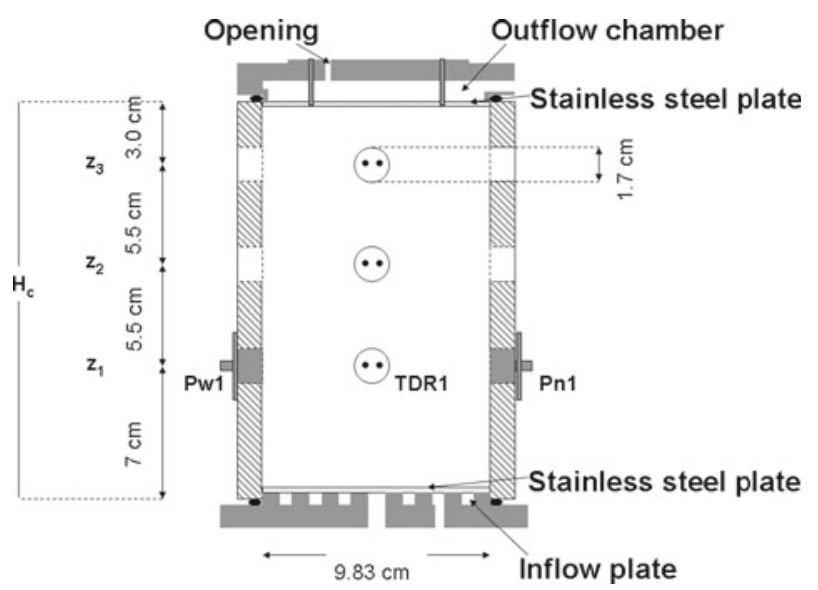

Fig. 2 Sand column set-up. Pressure transducers at elevation $z_{1}\left(P_{\mathrm{w} 1}\right.$ and $\left.P_{n 1}\right)$ are shown. Similar transducers were inserted at elevations $z_{2}$ and $z_{3}$

Table 1 Fluids properties at $20^{\circ} \mathrm{C}$

\begin{tabular}{llll}
\hline Properties & Water & PCE & Unit \\
\hline Density & 1000 & 1623 & {$\left[\mathrm{~kg} / \mathrm{m}^{3}\right]$} \\
Viscosity & $1 \times 10^{-3}$ & $0.9 \times 10^{-3}$ & {$[\mathrm{~Pa} \mathrm{~s}]$} \\
\hline
\end{tabular}

The two stainless-steel porous plates had a mean pore size of $40 \mu \mathrm{m}$ and were $3 \mathrm{~mm}$ thick. The bottom flow distribution plate, shown in Fig. 2, had channels in radial and tangential directions $(0.5-1.0 \mathrm{~cm}$ wide and $0.3-0.6 \mathrm{~cm}$ deep), which enabled uniform distribution of the invading or outgoing fluid. The plates had an intrinsic permeability of $3 \times 10^{-10} \mathrm{~m}^{2}$, which was much higher than that of the sand intrinsic permeability of $2.6 \times 10^{-10} \mathrm{~m}^{2}$. Contrary to many two-phase experiments, we did not use any hydrophilic and/or hydrophobic membranes. Such membranes can affect the distribution of fluids in the sand column.

Water and PCE pressures at various locations in the column were measured by means of selective pore pressure transducers. Standard pressure transducers (Kulite XTM190) were modified such that they were either hydrophobic or hydrophilic, making them suitable for measuring pressures of wetting or non-wetting phase, respectively. The face opening of the transducer in contact with soil had a diameter of $7 \mathrm{~mm}$. A full description of the transducers, their preparation for installation in the column, and their calibration are given in Bottero (2009) and in Oung et al. (2003).

Three pairs of pore pressure transducers were inserted at three different elevations $z_{1}, z_{2}$, and $z_{3}$ along the column (see Fig. 1). At each elevation, one wetting phase and one non-wetting phase transducer were inserted at the opposite sides of the column. The face of a transducer in contact with the soil was flush with the inner wall of the plexiglas column, so that transducer did not protrude into the soil.

A pressure transducer was inserted into the bottom inflow plate (see Fig. 1). In order to keep a desired constant non-wetting phase pressure at the bottom of the column the pressure transducer was connected to a pressure regulator which was placed at the top of the inflow burette, and regulated the pressure of the gas phase inside the burette (see Fig. 1). In this way, any pressure change at the bottom of the column was rapidly and automatically compensated by adjusting the pressure of the gas phase, restoring the bottom pressure to a preset value. 
The outflow chamber was kept at constant atmospheric pressure. This was achieved through a small opening $(0.5 \mathrm{~cm}$ diameter $)$ that was connected to a balloon filled with Argon gas. This set-up prevented any evaporation of water or volatilization of PCE while maintaining atmospheric pressure in the outflow chamber.

The local water saturation was measured inside the sand column using three time domain reflectometry (TDR) probes, placed at the same three elevations as the pore pressure transducers (see Fig. 2). Each TDR probe had two prongs, positioned in a horizontal plane with $1 \mathrm{~cm}$ spacing. This means that the measurement window of a TDR had almost the same size as a pressure transducer. TDRs were connected by $1 \mathrm{~m}$ long coaxial cables of $50 \Omega$ to a time domain reflectometry measuring device, TDR100 (Campbell Scientific Inc.), via coaxial multiplexer units SDMX50 (Campbell Scientific Inc). An algorithm was written in MatLab (www.mathworks.com) to control the multiplexer and to acquire the saturation as a function of time.

The overall fluid saturations of the whole sand column was determined separately from the change in the volume of fluids in inflow and outflow burettes. This change in volume was quantified by measuring the change in fluid pressure heads in the burettes. For this purpose, four differential pressure transducers (PDCR4160) were used, two placed in the inflow burette $\left(\mathrm{d} P_{1}\right.$ and $\left.\mathrm{d} P_{2}\right)$ and two in the outflow burette $\left(\mathrm{d} P_{3}\right.$ and $\left.\mathrm{d} P_{4}\right)$ (see Fig. 1). The differential pressures gave a measure of the change of volume of the two immiscible fluids. As there was no evaporation of water and/or volatilization of PCE, the volume change of any of the phases in the two burettes was assumed to be due to a corresponding change in the volume of that phase inside the sand column. This volume change was converted to saturation change for the whole column. Therefore, the overall water saturation of the column could be calculated as a function of time.

As mentioned earlier, no hydrophilic or hydrophobic membranes were used in our set-up. This means that once the invading fluid reached the outlet boundary, it flew out and the flow of both fluids continued until steady state was reached at a given imposed boundary pressure. For example, during drainage experiments, when the PCE breakthrough occurred, a large volume of wetting phase was still present in the column. Therefore, the outflow of both PCE and water continued until steady state was reached. At steady state, there was no flow of water, but, flow of PCE at a constant rate continued until we increased the imposed boundary pressure, and both fluids started to flow out again. This procedure meant that a large volume of PCE had to flow through the column during drainage experiments. In order to reduce the volume of PCE that was needed, a recirculation system was devised consisting of the two burettes, two pumps, and two on/off switches. A similar recirculation was applied during imbibition. On/off switches controlled both water and PCE pumps. Each switch consisted of two magnetic sensors, which were activated and deactivated when coming into contact with an aluminum floater inside the outflow burette (see Fig. 1). One floater and two magnetic sensors were placed in the lower side of the outflow burette to control the on/off switches of the PCE pump throughout the drainage processes. Another floater and two magnetic sensors were mounted in the upper side of the outflow burette to operate the switch for turning the water pump on and off during imbibition processes. In the inflow burette, a floater was placed on the surface of water to prevent contact between water and gas phases and therefore reduce the possibility of dissolution of air in water.

During drainage experiments, the non-wetting fluid was injected from the bottom of the column to avoid any instability due to gravity. PCE, after exceeding its entry pressure flowed upward in the sand column, displacing water. The outflowing water and later the outflowing PCE were collected in the outflow burette. Due to its higher density, the PCE sank to 
the bottom of the burette and was pumped back into the inflowing burette for continuous recirculation.

During imbibition experiments, water was provided into the top chamber of the column, and at the same time, the PCE pressure at the bottom plate of the column was reduced. When the PCE pressure was low enough, water entered the column from the top chamber and displaced PCE. After breakthrough, the water was collected in the outflow burette and pumped back into the inflow burette. The experiments were conducted in a constant-temperature room at $20^{\circ} \mathrm{C}$.

\section{Equilibrium Experiments}

The set-up described above was used to perform transient or equilibrium experiments. In transient experiments, the boundary phase pressures were chosen such that a full drainage (or full imbibition) of the column would occur in one step. Phase pressures and saturation were recorded as a function of time and analyzed in the framework of transient two-phase flow models. In the equilibrium experiments, drainage (or imbibition) of the column was performed in a series of transient experiments intermitted by equilibrium conditions, as explained below. During drainage experiments (primary and main drainage), PCE was injected into the sand column from below, by increasing its pressure in small increments (typically $300 \mathrm{~Pa}$ ). At each pressure step, the system was allowed to equilibrate over a 24-h period before increasing the pressure by the next increment. Although the time interval of $24 \mathrm{~h}$ was kept throughout the entire experiment, simulations showed that such a long period was required only when approaching irreducible water saturation. As mentioned above, at equilibrium, the receding phase was stagnant and the invading phase was either stagnant (during early parts of drainage) or flowed at a steady-state rate. At each equilibrium step, local pressures and saturations of both phases as well as the differential pressures in the inflow and outflow burettes were recorded.

A primary drainage experiment started with the column fully saturated with the wetting phase, i.e. water. Initially, the pressure of the non-wetting phase was increased until the entry pressure of the sand was reached. At that point, the non-wetting phase (PCE) flowed upwards into the sand column as a front, entering only the lower portion of the column. An equal volume of water exited the column at the top. Flow of both liquids decreased dramatically after a few hours. At the end of each equilibrium step, the wetting phase reached hydrostatic condition. The non-wetting phase also attained a linear pressure distribution in the stage before breakthrough. After breakthrough of the non-wetting phase, equilibrium was reached when PCE continued flowing through the column at a constant flow rate and water was stagnant at hydrostatic condition. The non-wetting phase pressure at the inflow plate was increased incrementally up to $12800 \mathrm{~Pa}$. During the last steps of pressure increase, no more water could be displaced out of the column, indicating that irreducible water saturation had reached. Additional increase of the non-wetting phase pressure resulted only in higher rates of PCE flow.

The primary drainage experiment was followed by the main imbibition experiment. At that point, the column was mainly filled with the non-wetting phase and water was at irreducible saturation. Imbibition was started by decreasing the pressure of the non-wetting phase at the bottom of the column in small increments and at the same time providing water continuously from above. The water phase was pumped at a constant rate from the outflow burette into the top chamber of the column. It should be noted that the top of the chamber was at atmospheric pressure and there was an overflow for water. Therefore, water provided at the top that did not enter the sand column flowed back to the outflow burette. Once the PCE pressure 
in the column became low enough, water entered the column, displacing PCE. This method prevented any instability caused by the density difference between the two phases. The PCE and water that later flowed out of the column were collected in the outflow burette and pumped back into the inflow burette. At the end of main imbibition, the column was mainly filled with water and PCE was at residual saturation. That formed the starting condition for main drainage. The procedure for the main drainage experiment was the same as for the primary drainage explained above.

\section{Locally Measured Pressure and Saturation}

Plot of fluids pressures and water saturation as a function of the equilibrium steps are shown in Fig. 3. Wetting phase pressures at the three elevations $z_{1}, z_{2}$, and $z_{3}$ are indicated by $P_{\mathrm{w} 1}, P_{\mathrm{w} 2}$, and $P_{\mathrm{w} 3}$, respectively. Non-wetting phase pressures at the same elevations are denoted by $P_{\mathrm{n} 1}, P_{\mathrm{n} 2}$, and $P_{\mathrm{n} 3}$. Figure 3 a shows the external non-wetting phase pressure measured at the bottom plate, as well as the wetting and non-wetting phase pressures in the sand column measured at three elevations $z_{1}, z_{2}$, and $z_{3}$ for a primary drainage experiment. The external pressure was increased incrementally, in steps of $300 \mathrm{~Pa}$, until a maximum pressure of $12800 \mathrm{~Pa}$. When PCE moved as a front, but before equilibrium was reached, water was also pushed out, and therefore, its local pressure increased during flow. However, once equilibrium was reached the water pressure decreased again to the hydrostatic value. That is why water pressures at the end of an equilibrium step, shown in Fig. 3a, are almost constant. The difference between $P_{\mathrm{w} 1}, P_{\mathrm{w} 2}$, and $P_{\mathrm{w} 3}$ is due to the hydrostatic pressure distribution. During flow, the pressure of the non-wetting phase at a given sensor increased dramatically as soon as PCE reached that sensor. Then, the internal non-wetting phase pressures increased incrementally, following the external pressure trend. All sensors had the same slope until pressure step 17, where breakthrough of the non-wetting phase occurred. At that point, there was a switch from a static equilibrium condition to a steady-state equilibrium condition, with a uniform flow of the non-wetting phase established.

Figure $3 \mathrm{~b}$ shows the local water saturation at equilibrium during primary drainage, as well as the overall water saturation of the column calculated from the volume changes in the inflow and outflow burettes. Water saturation started from 1, because the column was initially fully saturated with water, and decreased to the irreducible water saturation of 0.08 at $z_{1}$ and $z_{2}$ and to a higher water saturation of 0.16 at $z_{3}$, at the end of the primary drainage processes. As expected, the greatest water saturation was recorded at the uppermost sensor at $z=z_{3}$. The overall water saturation shows a much more gradual variation than the local saturations, in line with expectations.

The equilibrium local capillary pressure at a given elevation was calculated by subtracting the wetting phase pressure from non-wetting phase pressure measured at equilibrium. In Fig. 4a, the local capillary pressures at three elevations are plotted versus the local water saturations. In the same graph, the external pressure difference (i.e. the bottom non-wetting phase pressure minus the wetting phase pressure at top of the column) is plotted versus the overall column water saturation (the curve is denoted as $\Delta P-S_{\mathrm{w}}$ external). From the local capillary pressure, the entry pressure $P_{d}$ is found to be around $4000 \mathrm{~Pa}$. The same entry pressure was found by subtracting the hydrostatic pressure at the bottom of the column from the measured non-wetting phase pressure at the time of entry. The entry pressure found in this experiment is in disagreement with that reported by Hassanizadeh et al. (2004) for the same type of sand. They found an entry pressure of around $5800 \mathrm{~Pa}$. The capillary pressuresaturation relationship is assumed to be a property of the medium and fluids. Thus, the two 

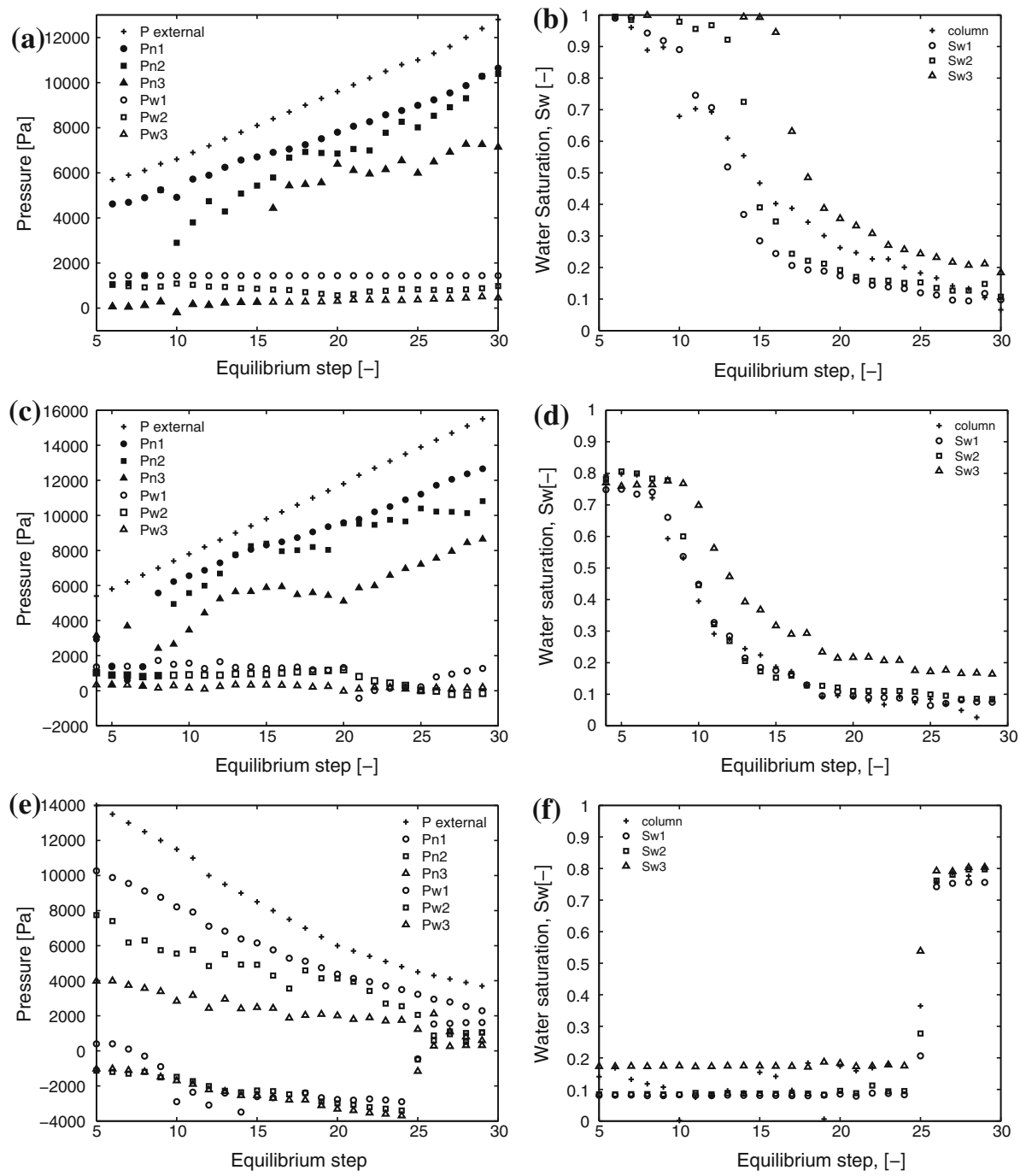

Fig. 3 a Non-wetting and wetting phase pressures along with the external pressure throughout primary drainage experiment; $\mathbf{b}$ Local water saturations and average water saturation versus equilibrium steps during primary drainage experiment; $\mathbf{c}$ Wetting and non-wetting pressures along with the external pressure throughout main drainage experiment; $\mathbf{d}$ Water saturation versus equilibrium steps in main drainage; e Local wetting and nonwetting phase pressures along with the external pressure throughout main imbibition experiment.; f Water saturation versus equilibrium steps in main imbibition

experiments are expected to provide the same parameters. However, it should be reminded that in their set-up, hydrophobic and hydrophilic membranes were placed at the top and at the bottom of the sample. Therefore, the question arises whether these membranes have had an effect on the capillary pressure-saturation relationship. In fact, we know that during drainage experiments, as soon as the non-wetting phase reaches a hydrophilic membrane, it starts to accumulate inside the porous medium. Therefore, for a given boundary pressure, the distribution of fluids in the soil is certainly affected by the membrane. How this affects the capillary pressure curves is not clear and needs to be investigated. 

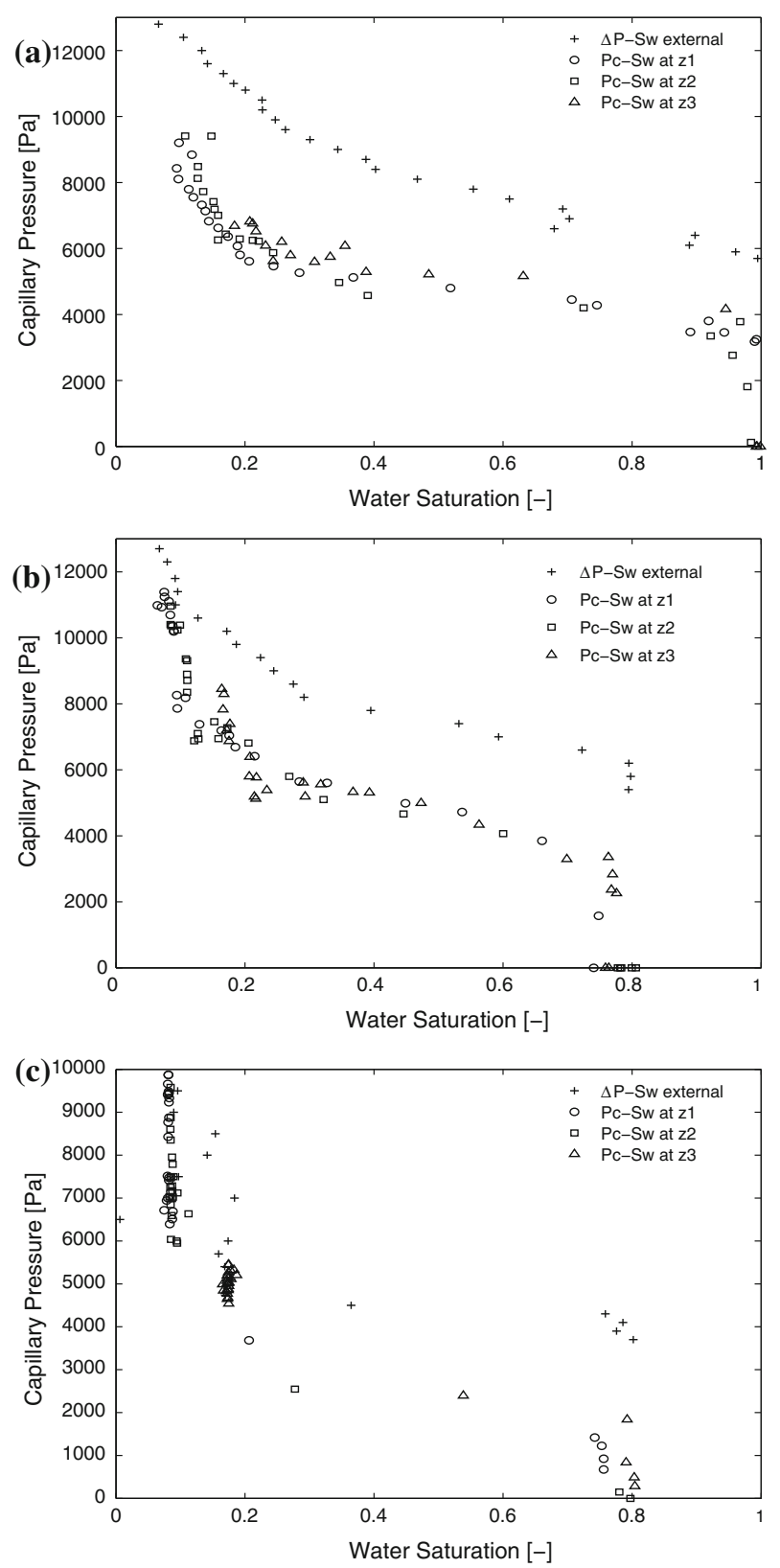

Fig. 4 a Local capillary pressure versus local water saturation at different elevations along with the external pressure versus average saturation throughout primary drainage; b Local capillary pressure versus water saturation along with the external pressure versus average water saturation in main drainage; cocal capillary pressure versus water saturation along with the external pressure versus average saturation during main imbibition experiment

The locally measured capillary pressure curves at three elevations coincide more or less, indicating that the column was reasonably homogeneous. It is clear that the external pressure difference (which is the same as the bottom non-wetting phase pressure because the wetting 
phase pressure at the top was zero) is significantly larger than the local capillary pressure values. The difference is mainly due to the effect of gravity as well as viscous forces when the non-wetting phase flows at steady state.

Figure $3 \mathrm{c}$ shows measured pressures of wetting and non-wetting phases during main drainage experiments. In the same graph, the external pressure of the non-wetting phase at the bottom of the column is shown. The pressure was increased incrementally in steps of 300 $\mathrm{Pa}$ until PCE residual saturation was reached at a maximum pressure of $15500 \mathrm{~Pa}$. Similar to the primary drainage experiment, the local non-wetting phase pressures have more or less the same trend as the external pressure until step 14 where the breakthrough of nonwetting phase occurred. We had expected that the water pressure would remain constant for all equilibrium points. This was the case for $P_{\mathrm{w} 3}$ sensor but not for sensors $P_{\mathrm{w} 1}$ and $P_{\mathrm{w} 2}$ which decreased significantly at step 21 . We believe that this occurred because the water phase became discontinuous in the lower half of the column as the irreducible water saturation was approached. Since PCE was injected from below, the water phase in the upper part of the column maintained continuity. This is also evident in Fig. 3d where saturation is plotted versus equilibrium steps; saturation at $z_{3}$ remained higher than the irreducible water saturation of 0.08 .

In Fig. 4b, the corresponding local capillary pressure versus local saturation and the external pressure difference versus the measured overall column saturation are plotted. Here, as for primary drainage, an entry pressure of around $4000 \mathrm{~Pa}$ was found. Also, the three local curves coincide quite well and the external curve shows a much larger capillary pressure values than the local value.

During main imbibition experiments, the external pressure of the non-wetting phase at the bottom was decreased incrementally from $15000 \mathrm{~Pa}$ to $4500 \mathrm{~Pa}$, in small steps of 400 and $200 \mathrm{~Pa}$. Figure $3 \mathrm{e}$ shows measured phase pressures versus equilibrium steps. Initially, wetting phase pressures measured by sensors showed negative values which was not expected. However, a hydrostatic distribution was attained when the imbibition front reached the corresponding wetting phase sensor at the equilibrium step 25, as shown in Fig. 3f. The negative pressure was probably due to the fact that the transducers were calibrated with continuous water phase, while water is discontinuous along the column. Under those conditions, the calibration of transducers is not valid. Local capillary pressure-saturation curves and the externally based capillary pressure curve are plotted in Fig. 4c. Here again, the same trends as for primary and main drainage curves are observed.

Primary drainage, main drainage, and main imbibition capillary pressure-saturation curves, obtained at measurement points $z_{1}, z_{2}$, and $z_{3}$, were fitted by Van Genuchten model (Van Genuchten 1980). For the fitting process, the capillary pressure $P_{\mathrm{c}}$ was considered as the independent variable and effective saturation $S_{\mathrm{e}}$ as the dependent variable:

$$
S_{\mathrm{e}}=\left[1+\left(\alpha P_{\mathrm{c}}\right)^{n}\right]^{m}
$$

where, the effective saturation $S_{\mathrm{e}}$ is expressed as:

$$
S_{\mathrm{e}}=\frac{S_{\mathrm{w}}-S_{r \mathrm{w}}}{1-S_{r \mathrm{w}}-S_{r \mathrm{n}}}
$$

Van Genuchten parameters were optimized by a trust-region algorithm (see Matlab, www.mathworks.com). Fitted parameters $\alpha$ and $n$ as well as the measure of goodness of fit $R^{2}$, are reported in Table 2. Figure 5 shows the comparison between fitted and measured $P_{\mathrm{c}}-S_{\mathrm{W}}$ curves. 
Table 2 Van Genuchten parameters obtained for primary drainage, main drainage, and main imbibition

\begin{tabular}{llll}
\hline VG parameters & Primary drainage & Main drainage & $\begin{array}{l}\text { Main } \\
\text { imbibition }\end{array}$ \\
\hline$n$ & 8.04 & 7.68 & 5.82 \\
$\alpha$ & $2.16 \times 10^{-4}$ & $2.06 \times 10^{-4}$ & $3.89 \times 10^{-4}$ \\
$R^{2}$ & 0.99 & 0.99 & 0.99 \\
\hline
\end{tabular}
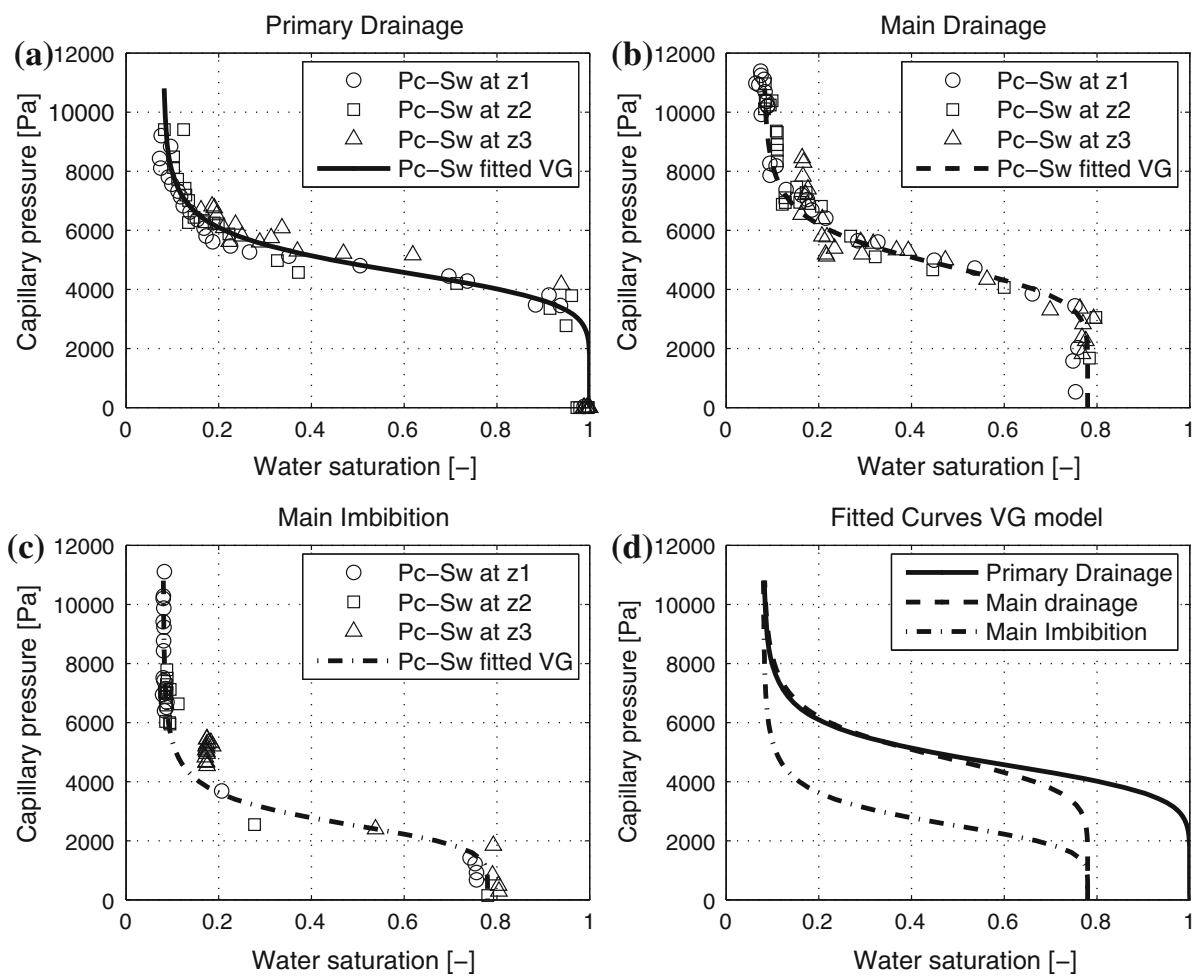

Fig. 5 Capillary pressure-saturation curves at elevation $z_{1}, z_{2}$, and $z_{3}$ along with the $P_{\mathrm{c}}-S_{\mathrm{W}}$ curve fitted by van Genuchten model: a Primary drainage; b Main drainage; c Main imbibition; $\mathbf{d}$ comparison between $P_{\mathrm{c}}-S_{\mathrm{W}}$ curves during primary drainage, main drainage, and main imbibition fitted by Van Genuchten model

\section{Averaging Operators}

The question arises how average pressures and water saturations should be calculated over the whole domain using local measurements. In this section, we introduce and compare different operators for average pressure. Definition of the column-scale average saturation is straightforward; it is the total volume of the $\alpha$ phase over the total pore volume of the column. Assuming constant porosity, this can be written in a discretized form as follows:

$$
<S_{\alpha}>=\frac{\sum_{j=1}^{N} S_{\alpha}^{j}}{N} ; \quad \alpha=\mathrm{w}, \mathrm{n}
$$


where the subscription $j$ denotes an observation point and $N$ represents the total number of observation points.

The definition of average phase pressure, however, is not straightforward. This issue has been recently discussed by Nordbotten et al. (2007, 2008) and Korteland et al. (2009). Korteland et al. (2009) introduced four averaging operators for pressure: simple average, simple phase-average, intrinsic phase-average, and centroid-corrected average. The simple average pressure is an arithmetic average of the phase pressure. The discretized form becomes:

$$
<P_{\alpha}>^{s}=\frac{\sum_{j=1}^{N} P_{\alpha}^{j}}{N} ; \quad \alpha=\mathrm{w}, \mathrm{n}
$$

In primary drainage experiments, the non-wetting phase is not always present everywhere within the sample. Nevertheless, a pressure is assigned to it everywhere. We assumed that the pressure of the non-wetting phase, when it is not present, is equal to the wetting phase pressure (i.e. we assumed capillary pressure to be zero). Usually, in many numerical codes (e.g. STOMP by White and Oostrom 1997; or MUFTE-UG, by Helmig 1997), when the nonwetting phase is not present, its pressure is defined to be equal to the wetting phase pressure plus the entry pressure. However, this assumption is quite arbitrary. One way of avoiding to introduce this assumption is to average the $\alpha$-phase pressure only within the region where the phase is actually present. We refer to this as simple phase-average pressure expressed as follows;

$$
<P_{\alpha}>^{\mathrm{sp}}=\frac{\sum_{j=1}^{N} P_{\alpha}^{j}\left(S_{\alpha}>0\right)}{N_{\left(S_{\alpha}>0\right)}} ; \quad \alpha=\mathrm{w}, \mathrm{n}
$$

This means that during primary drainage, when the non-wetting phase front advances along the domain, its pressure is averaged only over the region behind the front. At the early stage of the displacement, this can be just a small fraction of the whole domain. The water phase, however, is present everywhere, and its pressure is averaged over the entire domain. Therefore, the centroid of the non-wetting phase domain does not coincide with the centroid of the wetting phase domain or with the centroid of the averaging domain. The traditional way of averaging pressure is the intrinsic phase-average, where basically the microscale pressure of a phase is weighted by its volume fraction. Assuming constant porosity, the intrinsic phase-average can be written as follows:

$$
<P_{\alpha}>^{i}=\frac{\sum_{j=1}^{N} S_{\alpha}^{j} P_{\alpha}^{j}}{\sum_{j=1}^{N} S_{\alpha}^{j}} ; \quad \alpha=\mathrm{w}, \mathrm{n}
$$

Here also, the two phases are averaged over different domains and, therefore, the centroids of averaging domains are not the same.

In order to correct for this discrepancy in the centroids of average domains, Nordbotten et al. (2008) introduced another averaging operator, the centroid-corrected phase-average pressure which in one-dimensional form may be approximated as:

$$
\left[P_{\alpha}\right]=<P_{\alpha}>^{i}+\frac{1}{\frac{\partial}{\partial z}<z_{\alpha}>}\left(\bar{z}-<z_{\alpha}>\right) \frac{\partial}{\partial z}<P_{\alpha}>^{i} ; \quad \alpha=\mathrm{w}, \mathrm{n}
$$


where $\bar{z}$ is the centroid of the averaging domain and $\left\langle z_{\alpha}\right\rangle$ is the centroid of $\alpha$ phase, defined as:

$$
<z_{\alpha}>=\frac{\sum_{j=1}^{N} S_{\alpha}^{j} z_{\alpha}^{j}}{\sum_{j=1}^{N} S_{\alpha}^{j}} ; \quad \alpha=\mathrm{w}, \mathrm{n}
$$

Basically, in this averaging operator, the intrinsic phase-average pressure $\left\langle P_{\alpha}\right\rangle$ is corrected for the distance between the centroid of the averaging volume, $\bar{z}$, and the centroid of the phase, $\left\langle z_{\alpha}\right\rangle$. The derivatives of the intrinsic phase-average pressure and the position $<z_{\alpha}>$ can be calculated, for a vertical column with upward flow, as follows (Korteland et al. 2009):

$$
\begin{aligned}
\frac{\partial}{\partial z}<P_{\alpha}>^{i} & =\frac{1}{<S_{\alpha}>H}\left[-<P_{\alpha}>^{i}\left(S_{\alpha}^{\mathrm{top}}-S_{\alpha}^{\mathrm{bot}}\right)+\left(S_{\alpha}^{\mathrm{top}} \cdot P_{\alpha}^{\mathrm{top}}-S_{\alpha}^{\mathrm{bot}} \cdot P_{\alpha}^{\mathrm{bot}}\right)\right] \\
\frac{\partial}{\partial z}<z_{\alpha}> & =\frac{1}{<S_{\alpha}>H}\left[-<z_{\alpha}>\left(S_{\alpha}^{\mathrm{top}}-S_{\alpha}^{\mathrm{bot}}\right)+\left(S_{\alpha}^{\mathrm{top}} \cdot z_{\alpha}^{\mathrm{top}}-S_{\alpha}^{\mathrm{bot}} \cdot z_{\alpha}^{\mathrm{bot}}\right)\right]
\end{aligned}
$$

where $H$ is the length of the averaging domain, the superscripts "top" and "bot" refer to the values of the variable at the top and bottom of the averaging domain.

The average phase pressures and saturation are calculated based on the local measurements at three elevations $z_{1}, z_{2}$, and $z_{3}$. Moreover, an additional point at $z=z_{0}$ is considered, which is the lower boundary of the sand column (i.e. the top surface of the porous plate, see Fig. 2). The pressure of the non-wetting phase assigned to this virtual observation point is obtained from the external non-wetting phase pressure applied at the bottom of the column, as follows:

$$
P_{\mathrm{n} 0}=P_{\mathrm{ext}}-d_{0} \rho_{\mathrm{n}} g
$$

where $d_{0}$ is the thickness of the bottom porous plate. The wetting phase will have a hydrostatic pressure distribution and, thus, its pressure at $z_{0}$ is given as follows:

$$
P_{\mathrm{w} 0}=\rho_{\mathrm{w}} g H_{\mathrm{c}}
$$

where $H_{\mathrm{c}}$ in the length of the sand column. The water saturation at $z=z_{0}$ is calculated by using the van Genuchten model (Van Genuchten 1980). The averaging domain, therefore, is from $z_{0}$ to $z_{3}$, with a height $H=z_{3}-z_{0}$. Once average phase pressures are calculated, then the average capillary pressure is obtained by subtracting the average water pressure from the average non-wetting phase pressure.

\section{Reference Average Capillary Pressure}

In the previous section, various averaging operators were defined. The question arises which operator provides an average capillary pressure that is representative of the whole domain. An answer to this question can be found for the static stages, prior to breakthrough of the non-wetting phase. Under no-flow conditions, the total potential of each phase must be constant along the column. Therefore, the average total potential of a phase, $\left\langle\Phi_{\alpha}\right\rangle$, is simply the same as the local potential, $\Phi_{\alpha}$. Taking the bottom of the sand column $z_{0}$ as the reference elevation, the total potentials of PCE and water are, respectively: 


$$
\begin{aligned}
& <\Phi_{\mathrm{n}}>=\Phi_{\mathrm{n}}=P_{\mathrm{ext}}-\rho_{\mathrm{n}} g d_{0} \\
& <\Phi_{\mathrm{w}}>=\Phi_{\mathrm{w}}=\rho_{\mathrm{w}} g H_{\mathrm{c}}
\end{aligned}
$$

where $\rho_{\mathrm{n}}$ and $\rho_{\mathrm{W}}$ are the densities of the non-wetting and wetting phases, respectively, and $g$ is the gravity acceleration. Now, given the fact that an average pressure is assigned to the centroid of the averaging domain, then the correct average phase pressures under static conditions must satisfy the following relations:

$$
\begin{aligned}
& <P_{\mathrm{n}}>=P_{\mathrm{ext}}-\rho_{\mathrm{n}} g d_{0}-\frac{1}{2} \rho_{\mathrm{n}} g H \\
& <P_{\mathrm{w}}>=\rho_{\mathrm{w}} g H_{\mathrm{c}}-\frac{1}{2} \rho_{\mathrm{w}} g H
\end{aligned}
$$

These average phase pressures are here referred to as potential-based average pressures. The average capillary pressure is then calculated as follows:

$$
<P_{\mathrm{c}}>=<P_{\mathrm{n}}>-<P_{\mathrm{w}}>
$$

Thus, we consider the potential-based pressures, $\left\langle P_{\mathrm{n}}>\right.$ and $\left\langle P_{\mathrm{w}}>\right.$ as our reference pressure and use them to identify the correct average operator and the correct average capillary pressure.

\section{Results and Discussion}

Figure 6 shows average phase pressures, calculated using various operators, versus equilibrium steps during primary drainage. We notice significant differences between various averaging operators for the non-wetting phase pressure prior to breakthrough (around equilibrium step 17). After the breakthrough, however, all averaging operators yield more or less the same pressure. Note that the potential-based non-wetting phase pressure shows an overestimation after breakthrough. This is because then there is flow and there is viscous pressure drop which is not included in the calculation of potential-based pressure in Eq. 15. Before breakthrough, the simple average pressure lies below the other curves and below

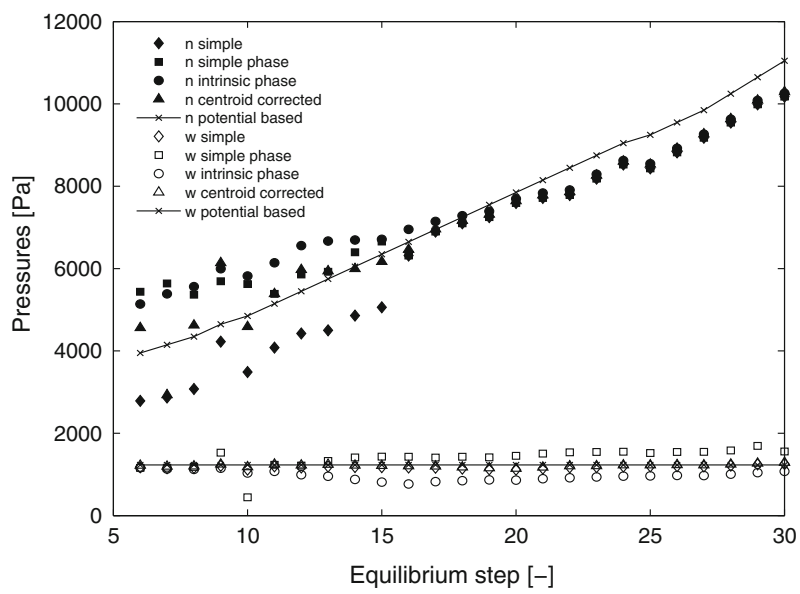

Fig. 6 Averaged non-wetting and wetting phase pressures versus equilibrium steps during primary drainage 
the reference pressure. This is due to the fact that with this operator, the non-wetting phase pressure is also averaged over the region above the front where the non-wetting phase is not present; so, we assume its pressure to be equal to the wetting phase pressure. In the case of simple phase-average operator, the average non-wetting phase pressure is calculated only over the domain behind the front. As a result, it overestimates the non-wetting phase pressure reference at the early stage of the front displacement. For example, when the front has just entered the column, then the simple phase-average pressure of the non-wetting phase is almost equal to the injection pressure (Fig. 6). Similarly, the non-wetting intrinsic phaseaverage pressure overestimates the reference curve because the high pressures in the lower part of the column are weighted by large values of non-wetting phase saturation. The centroid-corrected average pressure shows the best agreement with the potential-based average, albeit up until breakthrough. The centroid-corrected phase average pressure, as mentioned above, corrects the intrinsic phase average pressure for the distance between the centroid of the average domain $\bar{z}=H / 2$ and the centroid of each phase $\left\langle z_{\alpha}>\right.$. In fact, discrepancies among various averaging operators can be explained by the differences in the centroids of domains occupied by phases and the averaging domain. This is observed in Fig. 7 where the centroids of the wetting and non-wetting phases along with the centroid of the average domain are plotted versus equilibrium steps. It is clear that the centroid of the non-wetting phase moves up as the non-wetting front is at higher positions in the column at successive equilibrium steps. After the non-wetting phase breakthrough, and once the non-wetting phase saturation becomes almost uniform, its centroid reaches the middle of the averaging domain. At the beginning of the primary drainage, as the column is filled by water, the centroid of the water phase is the same as the centroid of the averaging domain. Then, when the non-wetting phase front moves upwards, the centroid of the wetting phase moves upwards too, as areas of higher water saturation are found at higher elevations. At non-wetting phase breakthrough, the centroid of the wetting phase will be at its highest position. Afterward, as the water saturation also becomes uniform, the water phase centroid moves down to approach the centroid of the averaging domain. However, because the water saturation at higher elevations remains

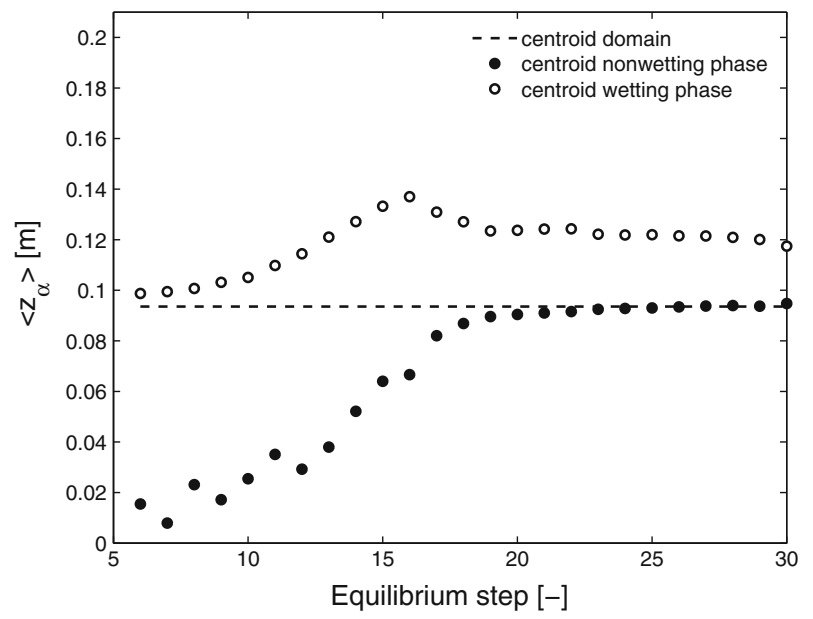

Fig. 7 Centroid of the average domain and centroids of the wetting and non-wetting phases versus equilibrium steps during primary drainage 


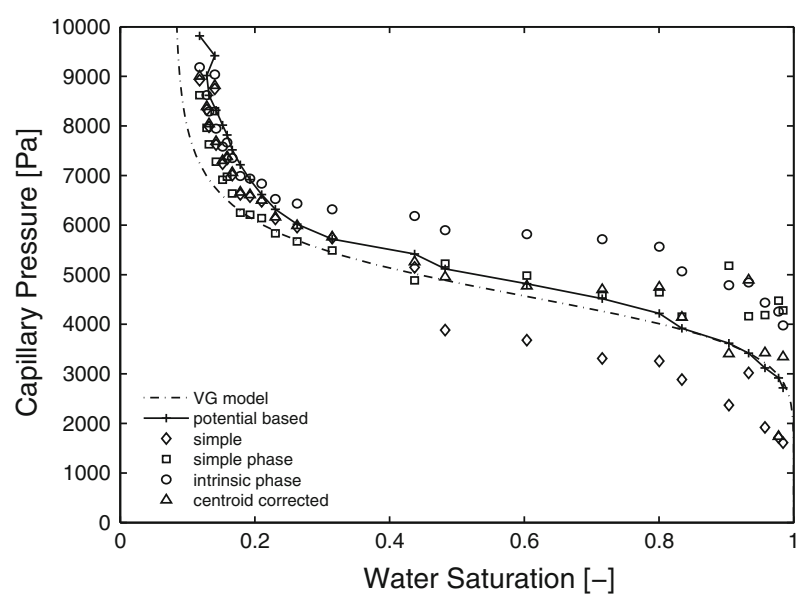

Fig. 8 Average $P_{\mathrm{c}}-S_{\mathrm{W}}$ curves along with the local one obtained by fitting $P_{\mathrm{c}}-S_{\mathrm{W}}$ data by Van Genuchten model. All curves refer to primary drainage

above irreducible value, the centroid of the wetting phase remains different from the centroid of the averaging domain.

In Fig. 8, the average capillary pressures, based on the four pressure averaging operators, are plotted versus the average wetting saturation $\left\langle S_{\mathrm{w}}>\right.$ and compared with the potential-based capillary pressure-saturation curve. It is evident that the centroid-corrected average approaches the reference curve or potential-based curve much better. Note that the potential-based average capillary pressure is valid only under static condition while the centroid-corrected holds also under flow condition (i.e after breakthrough). It should be noted that after the front reaches the end of the domain at water saturation $S_{\mathrm{w}}=0.4$, then simple average, simple phase-average and centroid-corrected average lie one above each other. Thus, at low water saturation, the differences between the various averages are not significant. Is is interesting to note that the curve based on intrinsic phase average systematically lies higher than the curves obtained by the other averaging operators. In Fig. 8, also the local capillary pressure-saturation curve is plotted. The comparison between the capillary pressure-saturation curve based on the centroid-corrected averaging operator with Van Genuchten model of locally measured curves (denoted by Van Genuchten model), shows that these two curves are close to each other. This we believe to be an indication of the homogeneity of the sand packing, as was also discussed in Sect. 4, where local curves measured at different elevations were found to coincide.

Various average pressures for main drainage experiments are shown in Fig. 9 where they are plotted versus equilibrium steps. Note that in the case of main drainage, both wetting and non-wetting phases are present in the whole domain, from the start. Therefore, the pressures obtained by the simple average and by the simple phase averaging operators are identical. It is clear that the differences between the average non-wetting pressures are much less pronounced than in the case of primary drainage. As both phases are always present, the centroids of domains domain occupied by phases and the averaging are now much closer to each other, as it can be seen in Fig. 10. In this case, the distances between the centroid of the average domain and the centroids of the two phases are smaller than in the case of primary drainage, see Fig. 7. Finally, in Fig. 11, capillary pressures based on simple average, intrinsic phase-average, and centroid-corrected average are plotted versus average wetting saturation 


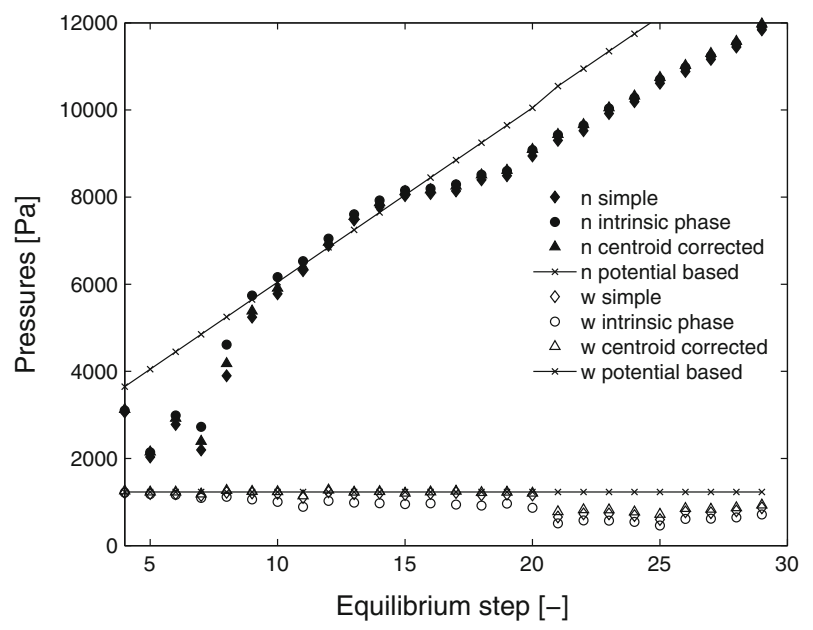

Fig. 9 Averaged non-wetting and wetting phase pressures versus equilibrium steps during main drainage

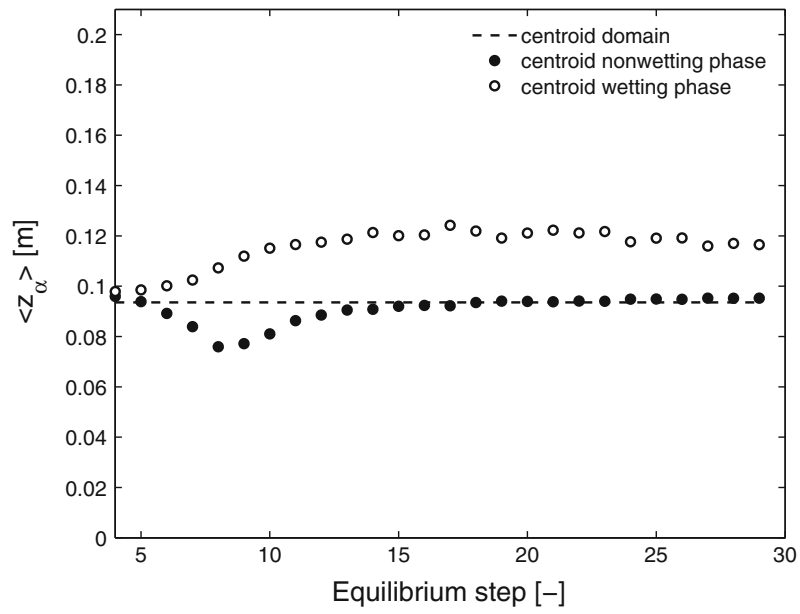

Fig. 10 Centroid of the average domain and centroids of the wetting and non-wetting phases versus equilibrium steps throughout main drainage

along with the reference $P_{\mathrm{c}}-S_{\mathrm{w}}$ curve. Also shown in Fig. 11 is the fitted local main drainage capillary pressure curve. It is evident that, all curves are in good agreement with the reference curve and with the local curve, except for the intrinsic phase average which lies just above them. Also, the comparison with the potential-based curve at low saturation is not appropriate as its definition is valid only under no-flow conditions, which is the case after breakthrough of PCE.

Curves of average pressure for main imbibition experiments are shown plotted against equilibrium steps in Fig. 12. The differences between various averages of non-wetting pressures and the potential-based pressure are not pronounced. A discrepancy, however, is seen between the potential-based pressure of water and the other average pressures. This is due to the fact that at the beginning of the imbibition process the column was mostly filled by the non-wetting phase with the wetting phase being at irreducible saturation. The water phase 


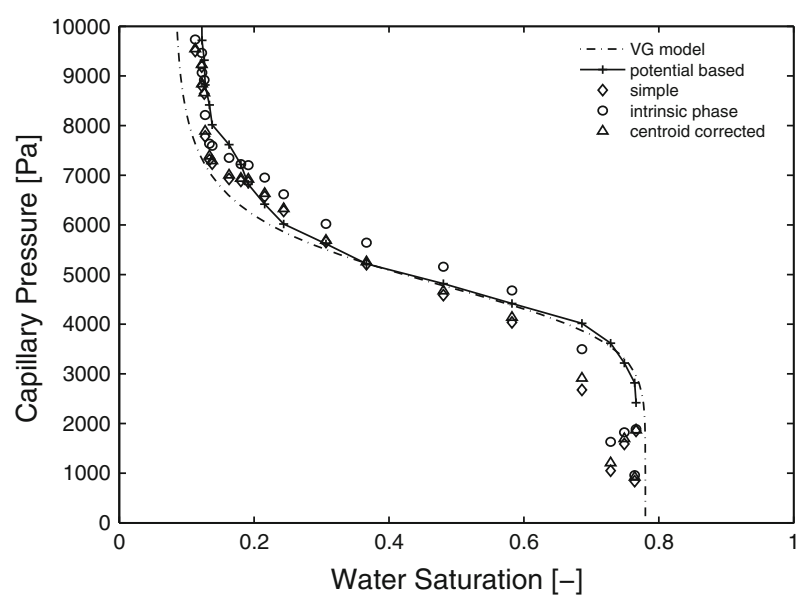

Fig. 11 Capillary pressure-saturation curves based on different averaging operators along with the reference curve and the local one. These refer to main drainage

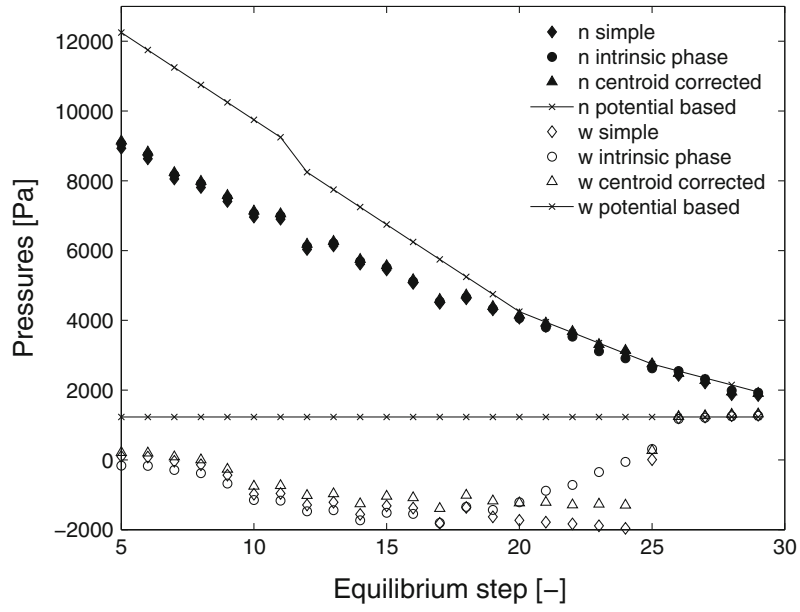

Fig. 12 Average non-wetting and wetting phase pressures during main imbibition

was discontinuous and transducers recorded negative values (see Fig. 3e). The applicability of the potential-based averaging operator requires a continuity of the phase along the domain which is not satisfied for the wetting phase. Thus, in this case, the comparison with the reference curve is not appropriate.

Figure 13 shows the centroid of wetting and non-wetting phases and the centroid of the averaging domain versus equilibrium steps. At the time that the wetting front reached sensor $z_{3}$ from above, the rest of the sand column was still mainly filled by the non-wetting phase, where the wetting phase was at irreducible saturation. Therefore, the centroid of the non-wetting phase was almost the same as the centroid of the averaging domain, while the centroid of the wetting phase was above because of the large wetting saturation around $z=z_{3}$. As the wetting front moved down into the averaging domain, the centroid of the wetting phase also moved downward. Once the front reached the bottom of the domain, the water phase was 


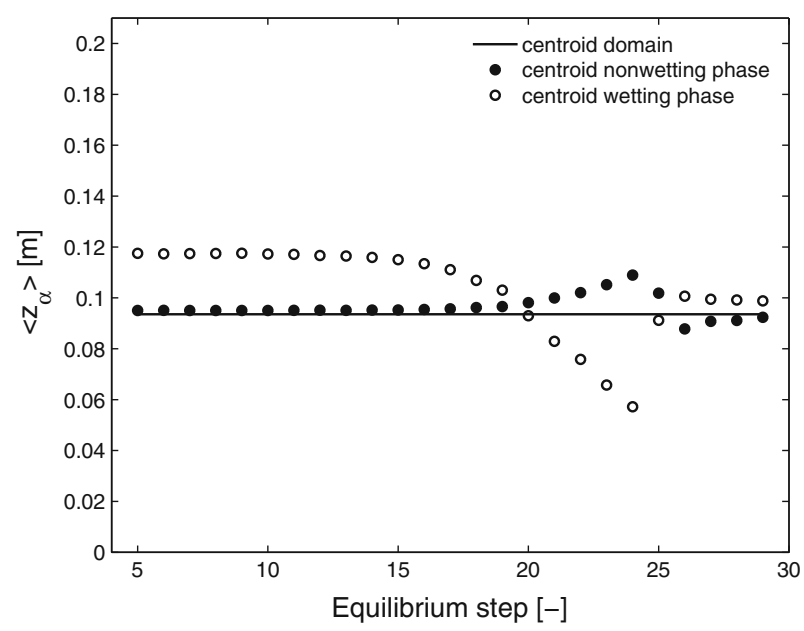

Fig. 13 Centroid of the average domain along with the centroid of the wetting and non-wetting phase versus equilibrium steps during main imbibition

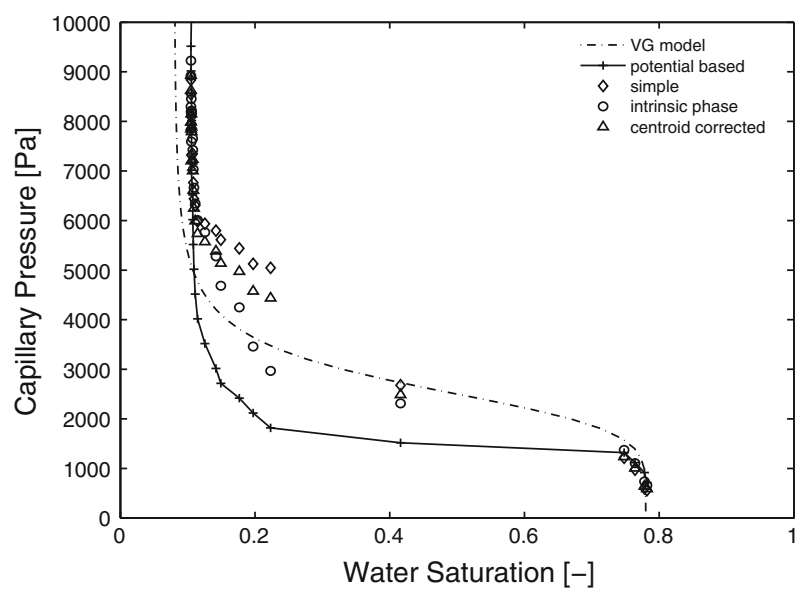

Fig. 14 Average capillary pressure-saturation curves obtained by different averaging operators along with the reference curve and with the local $P_{\mathrm{c}}-S_{\mathrm{W}}$. These regard main imbibition process

present everywhere at a high saturation (step 26) and its centroid approached the centroid of the average domain.

In Fig. 14, the averaged capillary pressure-saturation curves are plotted along with the local capillary pressure-saturation curve. For the sake of completeness, the potential-based average $P_{\mathrm{c}}-S_{\mathrm{w}}$ is also shown. However, one should be reminded that, because of the discontinuity of the water phase along the column, this curve is not really relevant here. It is evident that, contrary to drainage cases, there is not a really good agreement between any of the curves and the local fitted $P_{\mathrm{c}}-S_{\mathrm{w}}$ curve. It is interesting to note that the intrinsic phaseaverage $P_{\mathrm{c}}-S_{\mathrm{w}}$ curves lie below the curves obtained from all other averaging operators. As mentioned above, for the case of primary and main drainage, the $P_{\mathrm{c}}-S_{\mathrm{w}}$ curve from intrinsic phase-average pressure lies above the other curves. This behavior is reminiscent of dynamic pressure difference curves which for drainage lie higher and for imbibition lie lower, 
than $P_{\mathrm{c}}-S_{\mathrm{w}}$ curve. In other words, it seems that the use of the intrinsic pressure-average pressure will result in a pseudo non-equilibrium effect, even under equilibrium conditions. Therefore, this pressure averaging operator should not be used under any conditions.

\section{Summary and Conclusions}

A common procedure to determine capillary pressure is by subtracting the wetting phase pressure from the non-wetting phase pressure measured in fluid reservoirs external to the sand column. This is then assumed to be the representative average capillary pressure for the fluids-porous medium system. Such an external capillary pressure-saturation curve, however, is not representative of the system. This method is inaccurate because it does not account for the effects of gravity, specially for domains longer than a few centimeters. Instead, average capillary pressure should be determined from local pressure measurements and employing a more rigorous averaging procedure.

Drainage and imbibition experiments were performed under equilibrium conditions. Phase pressures and saturation inside the column, as well as external pressure and average saturation, were recorded at each equilibrium step. Various averaging operators were considered: simple average, simple phase-average, intrinsic phase-average, and centroid-corrected average. To establish which operator gives the correct average pressure, the results were checked against the requirement that total phase potential must be constant under no-flow conditions. This requirement allows the definition of a potential-based average pressure, which was considered to provide the true average pressure values under static conditions. Resulting potential-based average pressures were used to obtain reference $P_{\mathrm{c}}-S_{\mathrm{w}}$ curves.

During primary drainage, a large difference between non-wetting phase pressures, calculated by employing various operators, was obtained. The simple average operator averages the non-wetting phase also in the region ahead of the front where it is not actually present. As a consequence, the resulting non-wetting average pressure is an underestimation. The simple phase-average and the intrinsic phase-average operators both average the non-wetting phase only over the region where the phase is actually present. The intrinsic phase-average operator weights the local phase pressure by the phase saturation. In these two operators, the wetting and non-wetting phases are averaged over two different domains. Thus, the centroids of wetting phase and non-wetting phase do not coincide with each other and with that of the averaging domain. Both averages overestimated the reference average non-wetting phase pressure. The centroid-corrected average operator corrects the intrinsic phase-average pressure for the distance between the centroid of the domain and the centroids of the two phases. This was found to be in good agreement with the potential-based average pressure. After non-wetting phase breakthrough, both phases were present in the domain and the differences between pressures obtained by the various average operators were negligible. This is because the centroids of the two phases and the centroid of the averaging domain were close to each other. In the case of main drainage and imbibition, much less difference was found between various average of phase pressures, specially when both phases were present throughout the column. This is again because, under these conditions, the centroids of the phases and the averaging domain are close to each other. In general, the comparison between averaged capillary pressure-saturation curves has shown that the centroid-corrected average curves approached the potential-based $P_{\mathrm{c}}-S_{\mathrm{w}}$ curves for the cases of primary and main drainage. 
Open Access This article is distributed under the terms of the Creative Commons Attribution Noncommercial License which permits any noncommercial use, distribution, and reproduction in any medium, provided the original author(s) and source are credited.

\section{References}

Ahmadi, A., Quintard, M.: Large-scale properties for two-phase flow in random porous media. J. Hydrol. 183, 69-99 (1994)

Ataie-Ashtiani, B., Hassanizadeh, S., Oostrom, M., Celia, M., White, M.: Effective parameters for two-phase flow in a porous medium with periodic heterogeneities. J. Contam. Hydrol. 49, 87-109 (2001)

Ataie-Ashtiani, B., Hassanizadeh, S., Celia, M.: Effects of heterogeneities on capillary pressure-saturationrelative permeability relationships. J. Contam. Hydrol. 56, 175-192 (2002)

Bottero, S.: Advances in the theory of capillarity in porous media. PhD thesis, Utrecht University (2009)

Das, D., Hassanizadeh, S., Rotter, B., Ataie-Ashtiani, B.: A numerical study of micro-heterogeneity effects on upscaled properties of two-phase flow in porous media. Transp. Porous Media 56, 329-350 (2004)

Desbarats, A.: Upscaling capillary pressure-saturation curves in heterogeneous porous media. Water Resour. Res. 31(2), 281-288 (1995)

Eichel, H., Helmig, R., Neuweiler, I., Cirpka, O.: Upscaling of two-phase flow processes in porous media. In: Upscaling Multiphase Flow in Porous Media, pp. 237-257. Springer Verlag, Berlin (2005)

Gielen, T.: Dynamic effect in two-phase flow in porous media: a pore-scale network approach. $\mathrm{PhD}$ thesis, Delft University of Technology (2007)

Gielen, T., Hassanizadeh, S., Celia, M., Dahle, H., Leijnse, A.: A pore-scale network approach to investigate dynamic effects in multiphase flow. In: Proceedings of CMWRXV Conference, University of North Carolina (2004)

Gielen, T., Hassanizadeh, S., Nordhaug, H., Leijnse, A.: Dynamic effects in multiphase flow: a pore-scale network approach. In: Upscaling Multiphase Flow in Porous Media. Springer Verlag, Berlin (2005)

Hassanizadeh, S., Oung, O., Manthey, S.: Laboratory experiments and simulations on the significance of non-equilibrium effect in the capillary pressure-saturation relationship. In: Schanz, T. (ed.) Unsaturated Soils: Experimental Studies Proceedings of the International Conference: From Experimental Evidence towards Numerical Modeling of Unsaturated Soils, Weimar, Germany, September 18-19, 2003, vol. 1, pp. 3-14. Springer Verlag, Berlin (2004)

Helmig, R.: Multiphase Flow and Transport Processes in the Subsurface: A Contribution to the Modeling of Hydrosystems, p. 367. Springer, Berlin (1997)

Korteland, S., Bottero, S., Hassanizadeh, S., Berentsen, C.: What is the correct definition of average pressure? Transp. Porous Media (2009). doi:10.1007/s11242-009-9490-2

Manthey, S., Hassanizadeh, S., Helmig, R.: Macro-scale dynamic effects in homogeneous and heterogeneous porous media. Transp. Porous Media 58, 121-145 (2005)

Nordbotten, J., Celia, M., Dahle, H., Hassanizadeh, S.: Interpretation of macroscale variables in darcy's law. Water Resour. Res. 43, W08430 (2007). doi:10.1029/2006WR005018

Nordbotten, J., Celia, M., Dahle, H., Hassanizadeh, S.: On the definition of macro-scale pressure for multiphase flow in porous media. Water Resour. Res. 44, W06502. (2008) doi:10.1029/2006WR005715

Oung, O., Bezuijen, A.: Selective pore pressure transducers for use in model tests to study two-phase flow in porous media. Int. J. Phys. Model. Geotech. 4, 31-41 (2003)

Quintard, M., Whitaker, S.: Two-phase flow in heterogeneous porous media: the method of large-scale averaging. Transp. Porous Media 3, 357-413 (1988)

Van Genuchten, M.: A closed-form equation for predicting the hydraulic conductivity of unsaturated soils. Soil Sci. Soc. Am. J. 44, 892-898 (1980)

White, M., Oostrom, M.: STOMP-Subsurface Transport Over Multiple Phases. User's guide. Technical Report PNNL-11218, Pacific Northwest National Laboratory (1997) 